\title{
Genetic Variation and the Persistence of Predator-prey Interactions in the Nicholson-Bailey Model
}

\author{
Michael Doebeli \\ Zoology Institute, University of Basel, Rheinsprung 9, CH-4051 Basel, Switzerland
}

(Received on 29 October 1996, Accepted in revised form on 16 April 1997)

\begin{abstract}
The unstable Nicholson-Bailey predator-prey model is extended by assuming that the predation efficiency depends on quantitative characters in both the prey and the predator. The genetics of these characters is assumed to be determined by many diploid loci with additive effects. It is shown that in contrast to the monomorphic model, predator-prey coexistence is possible in the genetically variable system, and that even if the interactions lead to extinction, the time to extinction is typically much larger than in the monomorphic model. Coexistence is mediated by a coevolutionary arms race involving oscillations of the character means in the prey and predator populations. Such an arms race can occur even if costs to increase (or decrease) the character value are introduced, and coexistence is possible if such costs are not too high. The results also hold if self-limitation of the prey is included in the model. In this case, both the basic and the genetic model show the paradox of enrichment, but whereas predator-prey coexistence is only possible for low prey carrying capacities in the monomorphic model, the populations can persist in the genetically variable system even for very high prey carrying capacities. The results suggest that genetic variability is a condition which promotes predator-prey coexistence, and which should generally be taken into consideration when studying the ecology of extinctions.

(C) 1997 Academic Press Limited
\end{abstract}

\section{Introduction}

Simple ecological models serve important purposes. They lead to ecological paradigms, and when they fail to describe reality, they help identify missing theoretical components. For example, the LotkaVolterra competition equations lead to simple rules governing the coexistence of competing species, and these equations were the starting point for many attempts to identify mechanisms that prevent or promote competitive coexistence. Another example, which we will be concerned with in this article, is the classical Nicholson-Bailey host-parasitoid model, which was very influential for finding ecological conditions that can stabilize predator-prey interactions.

One general message emerging from the study of these models is that temporal and spatial variation in

E-mail: doebeli@ubaclu.unibas.ch. the parameters describing the ecological interactions promotes coexistence. These theoretical insights are supported by empirical work. An early example is Hutchinson's (1961) claim that the apparently paradoxical species richness in phytoplankton communities is possible because of temporal variation in physical conditions, which leads to continually changing competitive relations. For predator-prey interactions, Huffaker showed experimentally that a patchily distributed habitat can promote coexistence between two mite species that would both go extinct in a single habitat patch (Huffaker, 1958; Huffaker et al., 1963). More recent work by Holyoak \& Lawler (1996) showed that persistence of a protist predatorprey system is prolonged by asynchrony in the local dynamics of a metapopulation. In general, the role of spatial heterogeneity for population and community dynamics is a very active area of research.

Here I want to draw attention to the population dynamic effects of another source of variability. By 
using multi-locus genetics, I will investigate the effects of variation in a quantitative character on persistence in the unstable Nicholson-Bailey model. For this purpose, I will assume that quantitative characters in both the prey and the predator determine the rate at which prey is consumed by the predator. Since a certain amount of phenotypic and genetic variation in characters that determine ecological interactions is ubiquitous, this appears to be a natural extension. I will show that genetic variability reduces extinction probabilities and can lead to persistence in the Nicholson-Bailey model. Thus, one purpose of this paper is to argue that genetic variability is an important factor for the dynamics of ecological extinctions. The second purpose is to introduce the diploid version of the multi-locus genetic model for quantitative characters used in Doebeli (1996a, b, c).

\section{Model Description}

My starting point is the model of Nicholson \& Bailey (1935), which they used to study oscillations in predator-prey systems. The model is set in discrete time and has the following form. Let $N_{t}$ and $P_{t}$ be the prey and the predator densities at time $t$. Then,

$$
\begin{gathered}
N_{t+1}=\lambda N_{t} \exp \left[-a P_{t}\right] \\
P_{t+1}=b N_{t}\left(1-\exp \left[-a P_{t}\right]\right) .
\end{gathered}
$$

In the absence of the predator $\left(P_{t}=0\right)$, the prey is assumed to grow exponentially with a rate $\lambda$. The parameter $a$ is referred to as the searching or predation efficiency of the predator, and the term $\exp \left[-a P_{t}\right]$ is the probability that a prey individual escapes predation. It can be viewed as the 0th term of a Poisson distribution describing the number of encounters of a prey facing a population of $P_{t}$ predators with searching efficiency $a$ [for more details see e.g. Edelstein-Keshet (1987, p. 80)]. On the other hand, with probability $1-\exp \left[-a P_{t}\right]$ a prey individual is consumed and converted into $b$ predators. Nicholson \& Bailey (1935) introduced model (1) to study oscillations in a system in which a parasitoid controls the host population. Indeed, model (1) exhibits oscillations, as it is well known that there are no locally stable equilibria in system (1) (e.g. Murray, 1993). However, the size of these oscillations increases very rapidly, and the predator goes effectively extinct after a few generations (Fig. 1). Strictly speaking, it is easy to see that if $N_{t}$ and $P_{t}$ are both $>0$ in some generation, then so are $N_{t+1}$ and $P_{t+1}$ in the next generation, and hence that, deterministically, no extinction occurs in (1) if the system is started from an interior point in state space. However, even using high precision numbers, a computer will round the predator to zero after a few generations, and the prey will either also be set to zero or increase without bounds after the predator went extinct. Effectively, the rounding corresponds to setting a threshold below which populations are doomed extinct. All the results in this paper are based on numerical computer simulations, in which this threshold was set to $10^{-14}$. That is, whenever the predator density fell below this threshold, then the corresponding simulation run was stopped. For example, the simulation used for Fig. 1 was stopped after 22 generations.

In the sequel, I want to study the effect of variability in the searching efficiency $a$ on the persistence of the predator-prey system. I make the following assumptions. Prey individuals vary in a quantitative character $x$, and predator individuals vary in a quantitative character $y$. To have something specific in mind, think of these characters as size. I assume that for each prey phenotype $x$ there is a predator phenotype $y$ which optimizes the predation efficiency, and that this optimum changes linearly with the prey phenotype $x$. After a suitable transformation, we can then assume that $x$ and $y$ are measured in the same units, and that for each $x$ predation efficiency is maximised at $y=x$. Thus, I will assume that the searching efficiency is a symmetric function $a(x-y)$ of the difference $x-y$, and that $a(x-y)$ has a maximum at zero. In all that

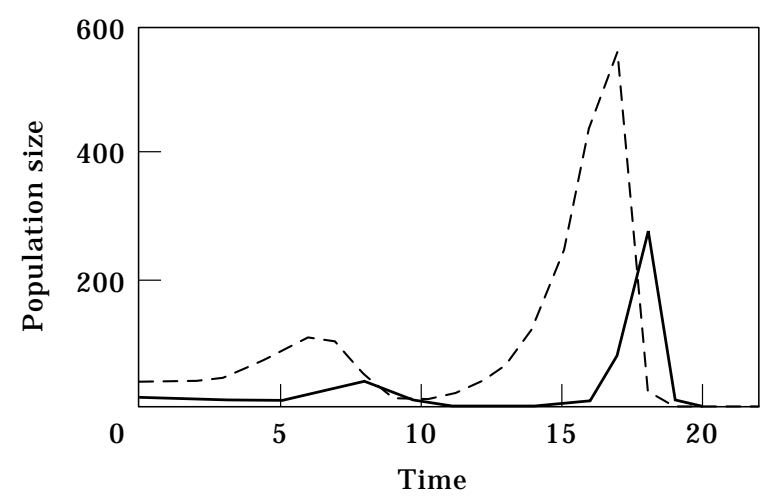

FIG. 1. Dynamics of the Nicholson-Bailey model. Model (1) was started from $N_{0}=40$ and $P_{0}=15$ and run until the predator essentially went extinct. For the graphic representation, population sizes in successive generations are joined by lines. For other initial conditions the trajectory would look different, but the time to extinction would be very similar (cf. also Fig. 3 ). The parameters in model (1) were $\lambda=2, a=0.05$ and $b=0.5$. Key: -, predator; -- , prey. 
follows, I assume that $a(x-y)$ is given by

$$
a(x-y)=a_{0} \exp \left[-\frac{(x-y)^{2}}{2 \sigma^{2}}\right]
$$

where $a_{0}$ is the maximal predation efficiency, and where $\sigma$ determines the "predation niche width", i.e. $\sigma$ is a measure of the range of prey phenotypes on which a particular predator phenotype is most effective. A small $\sigma$ implies a small predation niche width.

Let $n(x)_{t}$ denote the frequency distribution of the prey, i.e. $n(x)_{t}$ is the frequency of prey phenotype $x$ at time $t$. Similarly, let $p(y)_{t}$ denote the frequency distribution of the predator phenotypes at time $t$. Thus, if $N_{t}$ and $P_{t}$ are the total prey and predator densities at time $t$, then $n(x)_{t} N_{t}$ and $p(y)_{t} P_{t}$ are the densities of prey phenotype $x$ and predator phenotype $y$ at time $t$. Given $n(x)_{t}, p(y)_{t}, N_{t}$ and $P_{t}$, the task is to determine the corresponding quantities in the next generation $t+1$. For this I assume a two-step process in each generation: first, the ecological interactions due to predation take place, and then mating occurs in both the prey and the predator population. The first step is a straightforward extension of the basic model (1), and for the second step I will use an explicit diploid multi-locus model.

Let $\tilde{n}(x)_{t}$ and $\tilde{p}(y)_{t}$ denote the frequency distributions in the populations after selection given by the ecological interactions, but before mating. These quantities are determined as follows. For a prey phenotype $x$ the chance of escaping predation is the product of the probabilities of escaping from each predator phenotype $y$. Therefore,

$$
w(x)_{t}=\lambda \cdot \exp \left[\int_{y}-a(x-y) p(y)_{t} P_{t} \mathrm{~d} y\right]
$$

is the absolute contribution of prey phenotype $x$ to the next generation. The mean contribution (or mean fitness) at time $t$ is then

$$
\bar{w}_{t}=\int_{x} w(x)_{t} n(x)_{t} \mathrm{~d} x
$$

and it follows that we have

$$
\tilde{n}(x)_{t}=\frac{w(x)_{t}}{\bar{w}_{t}} \cdot n(x)_{t} .
$$

In addition, for the total prey population we have

$$
N_{t+1}=N_{t} \cdot \bar{w}_{t} .
$$

There is no expression corresponding to the fitness $w(x)_{t}$, eqn (3), for the predator in the Nicholson-Bailey model. Instead, the number of predator individuals of type $y$ after predation is just the sum of all converted prey individuals that are captured by this type of predator. In other words:

$$
\begin{aligned}
\tilde{p}(y)_{t} P_{t+1}=b \int_{x} n(x)_{t} N_{t}(1 & \\
& \left.\quad-\exp \left[-a(x-y) p(y)_{t} P_{t}\right]\right) \mathrm{d} x .
\end{aligned}
$$

The total predator density at time $t+1$ is simply

$$
P_{t+1}=\int_{y} \tilde{p}(y)_{t+1} P_{t+1} \mathrm{~d} y
$$

$=\int_{y} b \int_{x} n(x)_{t} N_{t}\left(1-\exp \left[-a(x-y) p(y)_{t} P_{t}\right]\right) \mathrm{d} x \mathrm{~d} y,(8)$

and the frequency $\tilde{p}(y)_{t}$ is obtained by dividing eqn (7) by eqn (8). Equations (3-8) thus yield the total population sizes $N_{t+1}$ and $P_{t+1}$, as well as the phenotype distributions $\tilde{n}(x)_{t}$ and $\tilde{p}(x)_{t}$ after selection through the ecological interactions.

We are left with describing the effects of mating, i.e. with providing a genetic recipe for determining the distributions $n(x)_{t+1}$ and $p(y)_{t+1}$ from the distributions $\tilde{n}(x)_{t}$ and $\tilde{p}(y)_{t}$. In order to use explicit multi-locus genetics, the phenotype space will be discretized into intervals measuring the additive effect of each allele. This means that integrals over phenotype space will be converted into appropriate sums. For the diploid genetic model, it is convenient to assume that the phenotype space, i.e. the range of possible character values $x$ and $y$, is an interval of the form $[0, c]$, where $c$ is an even positive integer. This does not imply any restrictions, since any other (finite) interval can be rescaled to such a choice. For the genetics of the quantitative characters $x$ and $y$ I assume that these characters are determined by $c / 2$ diploid loci, each with two alleles 0 and 1. The genome of an individual then consists of two strings of $0 \mathrm{~s}$ and $1 \mathrm{~s}$, and the corresponding character value is just the number of 1-alleles in the genome. Thus, an individual of phenotype $i$ has $i$-alleles in its genome, and phenotypes range from zero to $c$.

Suppose we are given two parent individuals with phenotypes $i$ and $j$, and suppose we know the distribution of offspring $q_{i, j}$ that results from a mating between $i$ and $j$. (Note that since we assume the same genetics in the prey and the predator, the distributions $q_{i, j}$ are the same for the prey and the predator.) To get the total offspring distribution, i.e. the distributions 
$n(x)_{t+1}$ and $p(y)_{t+1}$, one has to take a weighted sum over the distributions $q_{i, j}$, where the weights are the probabilities that a mating between phenotypes $i$ and $j$ occurs. These probabilities are determined by the phenotype distributions after selection, $\tilde{n}(x)_{t}$ and $\tilde{p}(y)_{t}$, since these distributions measure the contribution of each phenotype to the gamete pool. Assuming random mating leads to the following equations for the phenotype distributions in the prey and the predator at time $t+1$ :

$$
\begin{aligned}
& n(x)_{t+1}=\sum_{i, j} \tilde{n}(i)_{t} \tilde{n}(j)_{t} \cdot q_{i, j}(x) \\
& p(y)_{t+1}=\sum_{i, j} \tilde{p}(i)_{t} \tilde{p}(j)_{t} \cdot q_{i, j}(y) .
\end{aligned}
$$

Thus, we are left with determining the distributions $q_{i, j}$. Let $k$ be the number of loci in parent $i$ at which both genes are 1-alleles. I call $k$ the intragenomic overlap. Clearly, $k \leqslant i / 2$. At $i-2 k$ loci, there will be one 1-allele and one 0 -allele, and at the remaining loci there will be two 0 -alleles. Similarly, let $l \leqslant j / 2$ be the intragenomic overlap in the parent with phenotype $j$, i.e. the number of loci at which both genes are 1-alleles. An offspring of parents $i$ and $j$ with intragenomic overlaps $k$ and $l$ will have at least $k+l$ and at most $k+l+(i-2 k)+(j-2 l) 1$-alleles in its genome. Assuming Mendelian segregation and free recombination between loci, the probability $b(k, l)(z)$ that an offspring has $z$ 1-alleles, where $z$ is in the feasible interval $[k+l, k+l+(i-2 k)+(j-2 l)]$, is given by the binomial distribution

$b(k, l)(z)=\left(\begin{array}{c}(i-2 k)+(j-2 l) \\ z-(k+l)\end{array}\right) \cdot\left(\frac{1}{2}\right)^{(i-2 k)+(j-2 l)}$.

Clearly, the offspring distribution $q_{i, j}$ is now given as a weighted sum over the distributions $b(k, l)$, where the weights are the probabilities $\operatorname{prob}_{i, j}(k, l)$ that the parents' intragenomic overlaps are exactly $k$ and $l$. Since the parent genomes are independent, this probability can be written as the product

$$
\operatorname{prob}_{i, j}(k, l)=\operatorname{prob}_{i}(k) \cdot \operatorname{prob}_{j}(l)
$$

of the probabilities that parent $i$ has intragenomic overlap $k$ and parent $j$ has intragenomic overlap $l$. Thus:

$$
q_{i, j}=\sum_{k, l} \operatorname{prob}_{i}(k) \cdot \operatorname{prob}_{j}(l) \cdot b(k, l),
$$

and we are left with determining $\operatorname{prob}_{i}(k)$ for all $i$ and $k$. For a given phenotype $i$, the minimal possible intragenomic overlap is the maximum of the numbers 0 and $i-c$, and the maximal possible overlap is the smallest integer $t(i)$ which is $\leqslant i / 2$. Thus, $\operatorname{prob}_{i}(k)=0$ for $k \notin[\max (0, i-c), t(i)]$. Assuming no linkage, determining the remaining $\operatorname{prob}_{i}(k)$ is a simple exercise. There are $\left(\begin{array}{c}c \\ i\end{array}\right)$ possibilities to distribute $i$ 1 -alleles among the $c / 2$ diploid loci in the genome. We want to know how many of these result in exactly $k$ loci with two 1-alleles. There are $\left(\begin{array}{c}c / 2 \\ k\end{array}\right)$ possibilities to distribute these $k$ loci in the genome. We want to distribute the remaining $i-2 k 1$-alleles among the remaining $c / 2-k$ loci such that no locus has two 1-alleles. If we label the two genes at each locus with $\alpha$ and $\beta$, the possible ways to do this are to distribute $s$ of the $i-2 k$ 1-alleles among the $c / 2-k$ loci on the $\alpha$ gene, and the remaining $i-2 k-s$-alleles among the remaining $c / 2-k-s$ loci on the $\beta$ gene. Given $s$, the number of possible ways to do this is

$$
\left(\begin{array}{c}
c / 2-k \\
s
\end{array}\right) \cdot\left(\begin{array}{c}
c / 2-k-s \\
i-2 k-s
\end{array}\right)
$$

Summing over $s$ from zero to $i-2 k$, and multiplying by $\left(\begin{array}{c}c / 2 \\ k\end{array}\right)$, we obtain all possibilities of distributing $i$ 1 -alleles on $c / 2$ loci with exactly $k$ loci with two 1 -alleles. Dividing by $\left(\begin{array}{c}c \\ i\end{array}\right)$, we obtain the probability of intragenomic overlap $k$ in a individual with genotype $i$ as

$$
\begin{gathered}
\operatorname{prob}_{i}(k)=\left\{\left(\begin{array}{c}
c / 2 \\
k
\end{array}\right) /\left(\begin{array}{c}
c \\
i
\end{array}\right)\right\} \cdot \sum_{s=0}^{i-2 k}\left(\begin{array}{c}
c / 2-k \\
s
\end{array}\right) . \\
\left(\begin{array}{c}
c / 2-k-s \\
i-2 k-s
\end{array}\right) .
\end{gathered}
$$

Together with eqns (12), (10) and (9), this formula determines the genetic recipe to get the distributions $n(x)_{t+1}$ and $p(y)_{t+1}$ from the distributions $\tilde{n}(x)_{t}$ and $\tilde{p}(y)_{t}$, which in turn are obtained through the ecological interactions, eqns (3-8), from $n(x)_{t}$ and $p(y)_{t}$. The ecological and genetic equations together determine recursively the dynamics of the NicholsonBailey model with quantitative genetic variation in the predation efficiency.

\section{Results}

The basic effect of genetic variation in the predation efficiency is illustrated in Figs 2-6. In 

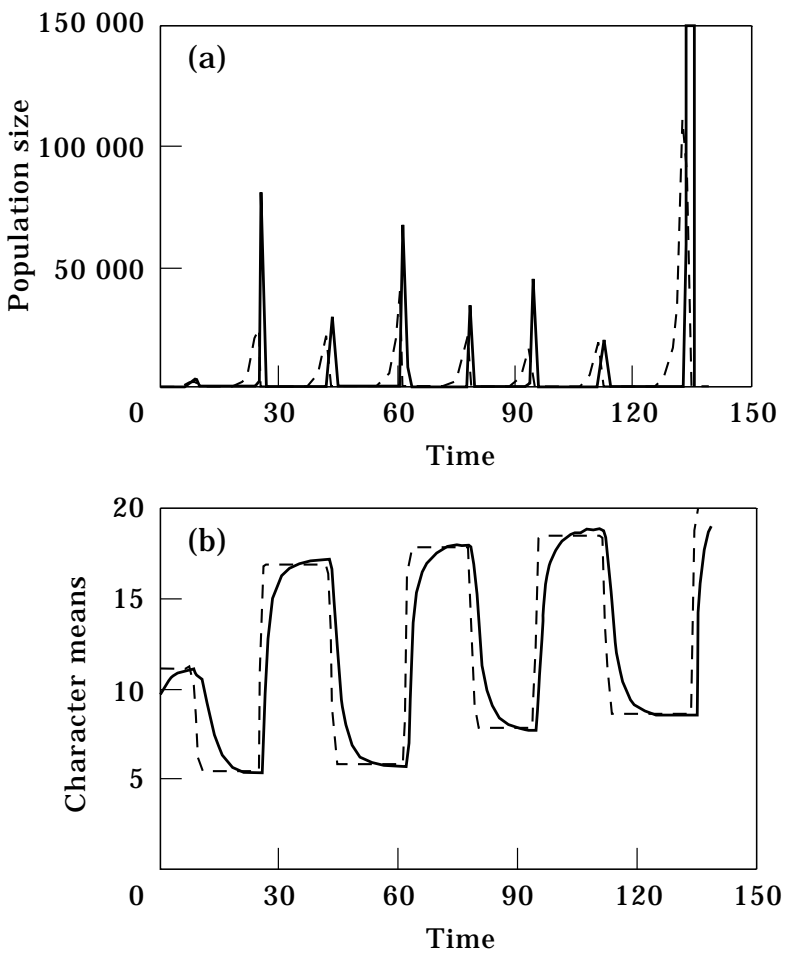

FIG. 2. Dynamics of the genetically variable Nicholson-Bailey model with ten loci. The parameter $\sigma$ in eqn (2) was set to $\sigma^{2}=6$, and the demographic parameters $\lambda, a$ and $b$ were the same as in Fig. 1. Figure 2(a) shows the time series of the prey and the predator population size, and Fig. 2(b) shows the time series of the character means in the prey and the predator. The interactions persist until generation 138. For different initial conditions the trajectories would look quantitatively different, but in general, persistence is greatly prolonged compared with the basic model (1). Key: -, predator; --, prey.

general, genetic variation prolongs the coexistence of predator and prey in the Nicholson-Bailey model. If the number of loci is not very large, the predator will still become extinct (Fig. 2). However, it typically takes much longer for this to happen than in the monomorphic model (1). Figure 2(b) illustrates the reasons for the prolonged persistence. In this figure, the character means in both the prey and the predator are plotted as a function of time. The predator is "chasing" the prey, which is trying to "escape", and as a consequence the means oscillate in the character interval. In the course of these oscillations, the means eventually come very close to the boundary of the character interval, which means that the two populations are essentially monomorphic and hence become extinct as in the basic model (1).

Figure 3 shows the distributions of the times to extinction for 1000 randomly chosen initial conditions in the monomorphic model (1) [Fig. 3(a)] and in the genetically variable model with nine loci [Fig. $3(\mathrm{~b})$ ]. It is apparent that genetic variation in predation efficiency greatly prolongs the time to extinction. Note that the distribution of times to extinction in Fig. 3(b) roughly follows a Poisson distribution, with some initial conditions leading to persistence over a very long period of time.

If the number of loci is large enough (Fig. 4), the oscillations of the means in the character interval are not big enough to hit the boundary of the character interval. Instead, these oscillations remain confined to the central part of the character interval [Fig. 4(b)]. As a consequence, the prey and the predator populations persist indefinitely, as suggested by numerical simulations.

The referee of this paper was concerned that the above results might be "knife-edge" results in the sense that the evolutionary arms race leading to cycling in phenotype space and to predator-prey coexistence might break down if costs to character states are introduced. Thus, if, say, having a high character value (e.g. a large size) has costs in terms of demographic parameters in both the prey and the
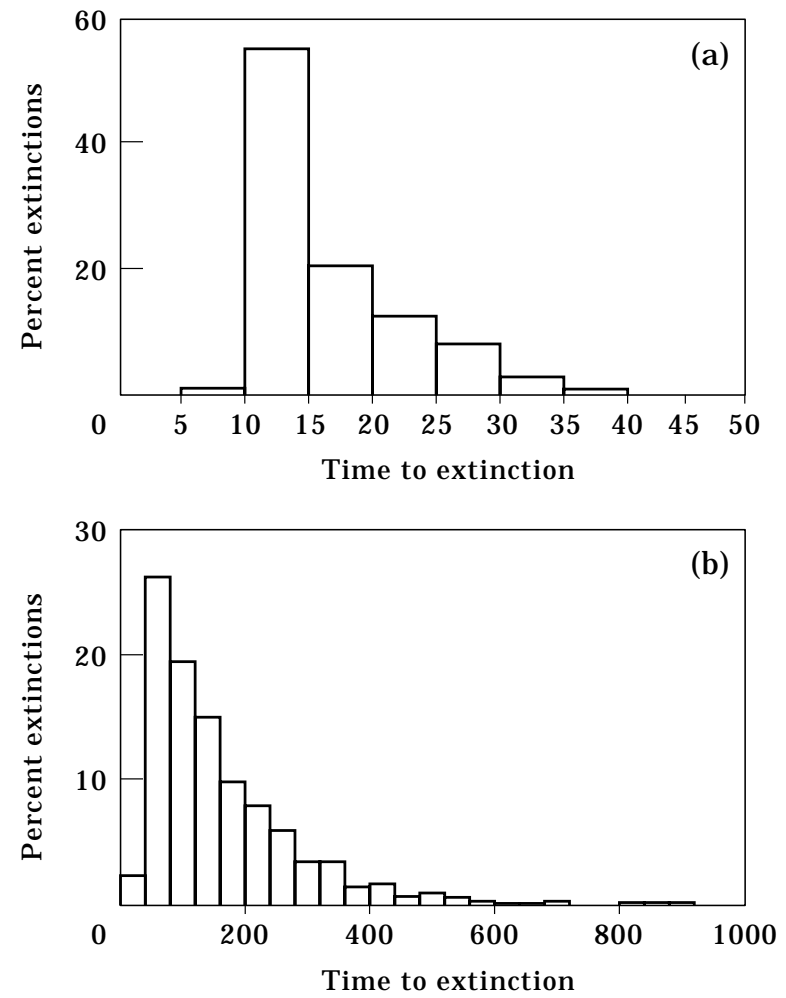

FIG. 3. Distribution of the times to extinction in the monomorphic [Fig. 3(a)] and in the polymorphic model [Fig. 3(b)]. For 1000 randomly chosen initial conditions, the system was run until the predator went extinct, and the time to extinction was recorded. The panels show percentages of times to extinction falling into intervals separated by five time steps [Fig. 3(a)] and by 40 time steps [Fig. 3(b)]. The demographic parameters were the same as in Fig. 1. In addition, I set $\sigma^{2}=6$ in the polymorphic model, in which the number of loci was nine. 

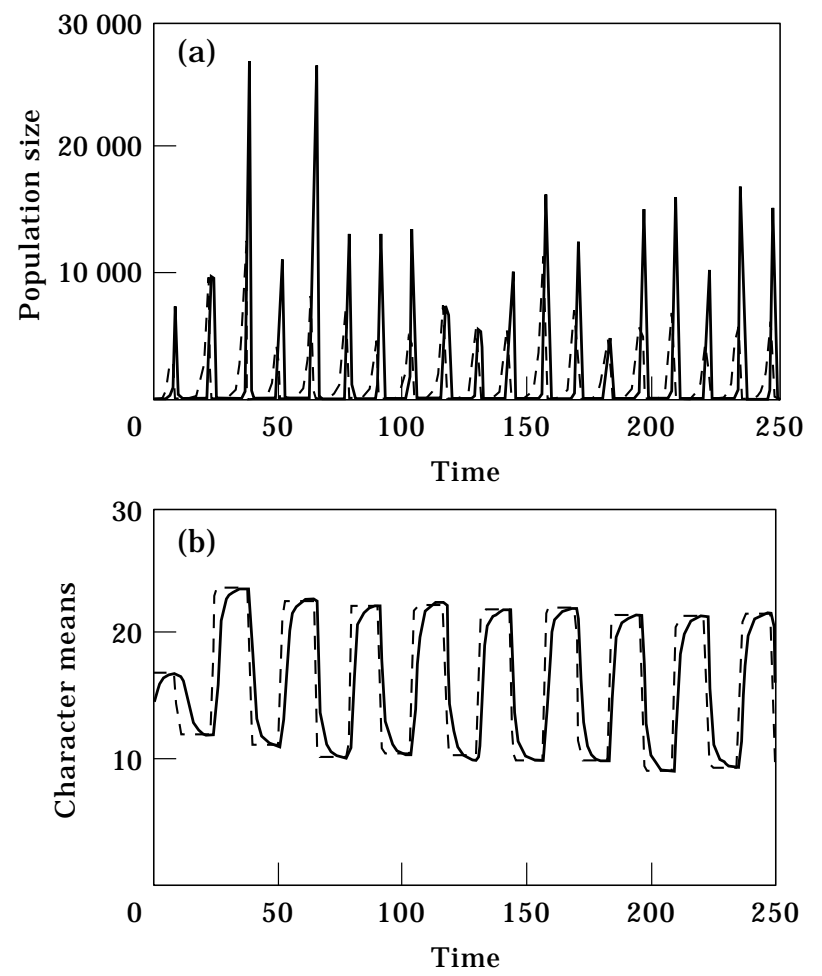

FIG. 4. Persistence in the genetically variable Nicholson-Bailey model. Everything is the same as in Fig. 2, except that the number of loci was increased to 15 . In this case, numerical simulations suggest that no initial conditions lead to extinction of the populations. Key: -, predator; --, prey.

predator, evolution towards higher character values might be impossible, which would cut the increasing branches of the phenotypic oscillations shown in Figs 2(b) and 4(b). I have tested this prediction by including costs in the model as follows. In the prey, instead of working with a fixed intrinsic growth rate $\lambda$ in eqn (3), I assumed that the growth rate depends linearly on the character:

$$
\lambda(x)=\lambda_{0}-r \cdot x .
$$

Here $\lambda_{0}$ is the maximal growth rate (attained at the smallest character value 0 ), and $r>0$ measures the cost of having character value $x$, where $x \in[0,2 c]$, with $c$ the number of loci. Similarly, in the predator I assumed that the conversion efficiency $b$ in eqn (7) depends linearly on the character state:

$$
b(y)=b_{0}-s \cdot y,
$$

where $b_{0}$ is the maximal efficiency, and $s>0$ determines the cost to a predator having character value $y \in[0,2 c]$. Thus, I assumed that an increased character state has a cost in terms of demographic parameters in both the prey and the predator. (Note that the opposite assumption of a decreased character state inducing a cost would yield the same results, because of the intrinsic symmetry in the genetic model without costs, which is due to the fact that for the predator-prey interactions only similarity in character states matters, and not whether similarity occurs between individuals with high or between individuals with low character values.)

Typical results from the model including costs are shown in Fig. 5, for which the number of loci and the maximal predation efficiency was the same as in Fig. 4 , but costs were added using eqns (15) and (16). In Fig. 5(a), the means in the prey and the predator are shown as a function of time, with $\lambda_{0}=2.6$ and $r=0.04$ in eqn (15), and $b_{0}=0.7$ and $s=0.013$ in eqn (16). These values were chosen such that the intrinsic prey growth rate varies between 2.6 and 1.4 and the conversion efficiency varies between 0.7 and 0.3 , and such that the intermediate phenotypes $c$ had the same demographic parameters as used for Fig. 4. As in that case, and despite the costs, there is indefinite persistence of the predator-prey interaction, which is again mediated by persistent evolutionary oscillations of the characters. Even though increasing the character state incurs a cost, the prey "escapes" from low character values towards higher values if the predators have a character mean that is close to that of the prey; the disadvantage of higher character values is offset by the advantage of being dissimilar from the predator. In turn, the mean predator character also increases and follows the prey character, because the disadvantage of having higher character values is offset by the advantage of being similar to the prey. Due to the cost, the phenotypic oscillations are more irregular and not as symmetric as those in Fig. 4(b) with respect to the midpoint of the character interval. Instead, the oscillations are slanted towards the lower part of the character interval. Nevertheless, they are confined to the interior of the character interval, which permits persistence.

If costs are very high, the slant in the oscillations toward lower character values becomes more pronounced. Moreover, the system can get thrown off balance because different regions of the character interval correspond to quite different demographic parameters and hence to different oscillatory regimes in the basic Nicholson-Bailey model (1). For example, in Figs 5(b) and (c), due to the costs the character means eventually get quite close to the boundary of the character interval, which on the one hand means that the system approaches the monomorphic model (1), and on the other hand leads to demographic parameters that induce very large fluctuations (see also remarks below). As a consequence, the subsequent increase in character states 
and restoration of polymorphy are not sufficient for the predator to recover from the previous density crash, and extinction results. Due to the imbalance resulting from different demographic regimes being associated to different character states when there are costs, the character means need not come as close to
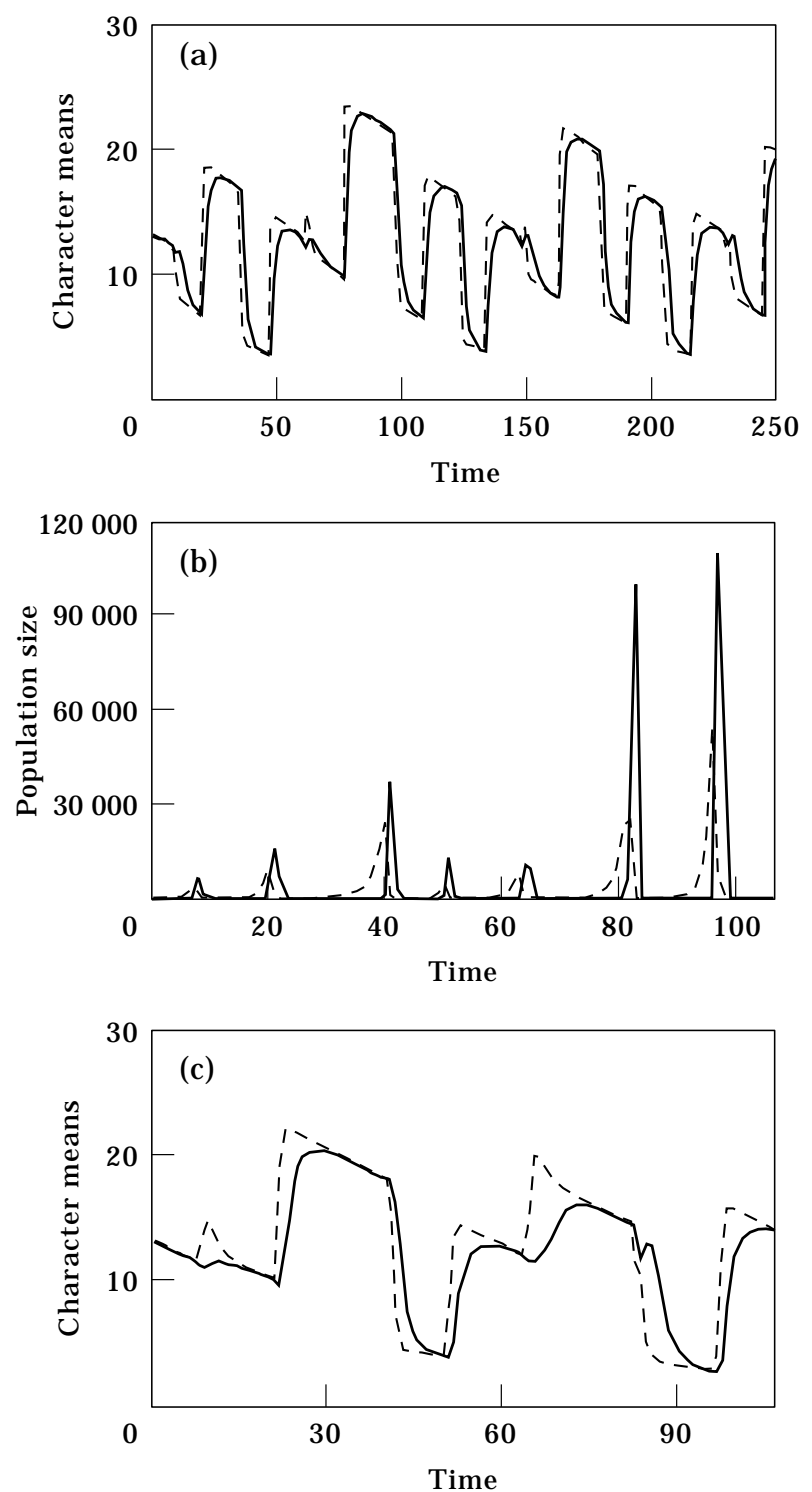

FIG. 5. Effects of costs to increased character states on persistence. Figure 5(a) is the same as Fig. 4(b), except that eqns (15) and (16) were used with $\lambda_{0}=2.6, r=0.04, b_{0}=0.7$ and $s=0.013$. Numerical simulations starting from different initial conditions suggest indefinite persistence. Figs 5(b) and (c) are the same as Figs. 4(a) and (b) except that eqns (15) and (16) were used with $\lambda_{0}=2.9, r=0.06, b_{0}=0.95$ and $\mathrm{s}=0.03$. These values imply that the intrinsic prey growth rate $\lambda(x)$ varies between 2.9 and 1.1, and the predator conversion efficiency $b(y)$ varies between 0.95 and 0.05 , with the intermediate phenotypes $c$ having the same demographic parameters as assumed for Fig. 4. In the simulation run shown, the predator went extinct after ca. 100 generations. Key: -, predator; --, prey. the boundary of the character interval as without costs for extinction to occur [cf. Fig. 2(b)], and costs can lead to extinction where no costs would allow persistence. We note, however, that with the costs used for Fig. 5(b) and (c), persistence is possible if the number of loci is increased enough.

Figure 5 illustrates the general fact that while costs affect the basic results from the models without costs quantitatively, these results are qualitatively robust against introducing costs. That is, even if there are differential costs for different character states, genetic variability reduces extinction probabilities and can lead to predator-prey coexistence through persistent evolutionary cycling in phenotype space. Similar conclusions hold if other, nonlinear cost functions are used, and if other combinations of demographic parameters (e.g. $\lambda$ in the prey, maximal predation efficiency $a_{0}$ in the predator) are used to implement the costs. Moreover, numerical experiments show that the remaining results presented below are also qualitatively robust against introducing costs to character states.

In the genetic model without costs, the predation niche width determined by $\sigma$ in eqn (2) is a parameter that is in a sense complementary to the number of loci. Decreasing $\sigma$ corresponds to decreasing the range of prey phenotypes over which a particular predator phenotype is most effective. Decreasing the predation niche width leaves more room in phenotype space for the prey to escape a particular predator phenotype and therefore has the same effects as increasing the number of loci in the system. This phenomenon is illustrated in Fig. 6, where the dynamics of the system with the same number of loci as in Fig. 4 are plotted in the vertical direction for different values of $\sigma$. For each value of $\sigma$, the system was started from the same initial conditions and run for 250 generations before the next 50 generations were plotted on the $y$-axis. As $\sigma$, and hence the predation niche width increases, the amplitude of the predator-prey cycles increases, and for large enough values of $\sigma$ the system does not persist and goes extinct before generation 250. In view of Fig. 3, Fig. 6 may look quantitatively different if different initial conditions were used. Nevertheless, Fig. 6 captures the general effect of increasing $\sigma$, i.e. the predation niche width.

Figures 2-4 and 6 show the general effect of increasing the number of loci and of increasing the parameter $\sigma$. The other three parameters in the system, the prey growth rate $\lambda$, the conversion coefficient $b$ and the maximal predation efficiency $a_{0}$, only quantitatively affect these outcomes. The following two general trends capture the main effects 

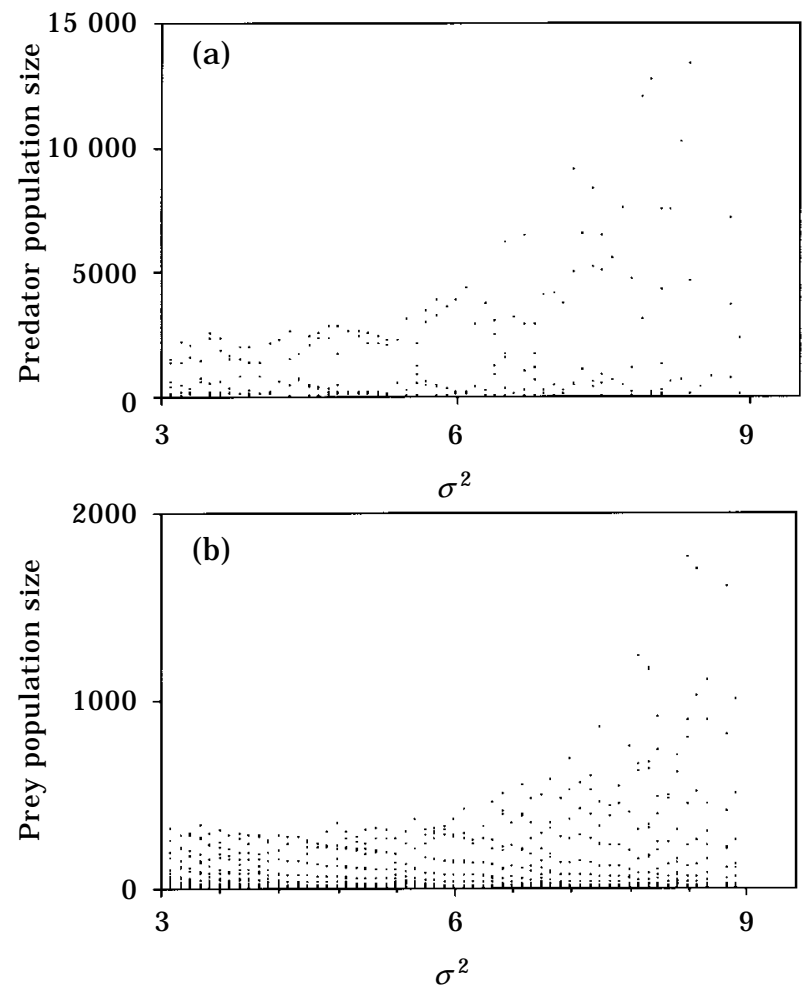

FIG. 6. Dependence of the predator-prey dynamics on the predation niche width determined by the parameter $\sigma$ in eqn (2). The same demographic parameters and the same number of loci as in Fig. 4 were used. As $\sigma$ and therefore the predation niche width is increased, the size of the population oscillations in the predator [Fig. 6(a)] and in the prey [Fig. 6(b)] increase. For 60 equally spaced values of $\sigma^{2}$, the system was run for 250 generations, and then the next 50 generations were plotted in the vertical axis. For higher values of $\sigma$, the predator is at very low densities for most of the time, with occasional excursions to very high densities. For values of $\sigma^{2} \geqslant 9$, the system did not persist until generation 250 .

of these parameters as seen in extensive numerical simulation: predator-prey persistence is most likely for low to intermediate prey growth rates $\lambda$, and for intermediate ratios $b / a_{0}$ (the exact quantitative meaning of "intermediate" depends on the other parameters in the system).

One way to stabilize the monomorphic model (1) is to include self-limitation in the prey, which is typically done (Beddington et al., 1975) by using a Ricker type density dependence in the prey, i.e. by replacing the first equation in (1) by

$$
N_{t+1}=N_{t} \lambda \cdot \exp \left[-\frac{\ln \lambda}{K} N_{t}\right] \cdot \exp \left[-a P_{t}\right] .
$$

Here $K$ is the carrying capacity of the prey, that is, in the absence of the predator, $K$ is an equilibrium for the prey dynamics. This equilibrium may be unstable, but here I concentrate on the case where it is locally stable. (As is well known and easy to see, this is the case if and only if $\ln \lambda<2$.) Thus extended, the Nicholson-Bailey model shows a form of the paradox of enrichment (Rosenzweig, 1971; Hassell, 1978): for low carrying capacities, the full predator-prey system has a stable equilibrium, which gives way to oscillations of increasing magnitude as the carrying capacity $K$ is increased. If the carrying capacity of the prey is too large, the predator prey system cannot persist, which leads to the paradox. The phenomenon is shown in Fig. 7(a).

It is straightforward to include self-limitation of the prey in the genetically variable model by replacing the phenotypic fitness functions $w(x)_{t}$, eqn (3), of the prey by

$$
\begin{aligned}
& w(x)_{t}= \\
& \lambda \cdot \exp \left[-\frac{\ln \lambda}{K} N_{t}\right] \cdot \exp \left[\int_{y}-a(x-y) p(y)_{t} P_{t} \mathrm{~d} y\right],
\end{aligned}
$$

where $N_{t}$ is the total prey density at time $t$. That is, I assume that all prey phenotypes are affected equally by density dependent effects in the prey population. The genetically variable system still exhibits the paradox of enrichment in that increasing the prey carrying capacity leads to predator-prey oscillations of increasing magnitude. However, the predator-prey system shows persistence even for very large prey carrying capacities. This is illustrated in Figs 7(b) and 7(c). In Fig. 7(b), the behaviour of the multi-locus system is shown for the same range of prey carrying capacities as in Fig. 7(a). While predator-prey coexistence only starts at slightly larger carrying capacities than in the monomorphic model, the regions of coexistence at a stable equilibrium are of similar size. As the systems become unstable, the fluctuations in the variable system are larger, because the total carrying capacity of the prey, summed over all phenotypes, is larger than in the monomorphic system, which increases the variance in the prey population size and hence the fluctuations. However, the variable system persists for prey carrying capacities for which the predator in the monomorphic model goes extinct $(K \geqslant 74)$. This effect is rather large, as is shown in Fig. 7(c): the genetically variable predator-prey system persists for carrying capacities up to $K \sim 6800$. While the system seems to persist indefinitely for all values in Fig. 7(b), the exact numerics for Fig. 7(c) may depend quantitatively on the initial conditions. For Fig. 7(c), the same initial conditions were used for each value of $K$, and then the system was run for 400 generations before the next 50 generations were plotted for each $K$. In view of the considerable variance in times to extinction (cf. Fig. 
3), values of $\mathrm{K}$ indicating persistence in Fig. 7(c) may lead to extinction before generation 400 if different initial conditions were used, while values of $K$ leading to extinction (e.g. $K \sim 4500$ ) may indicate persistence.
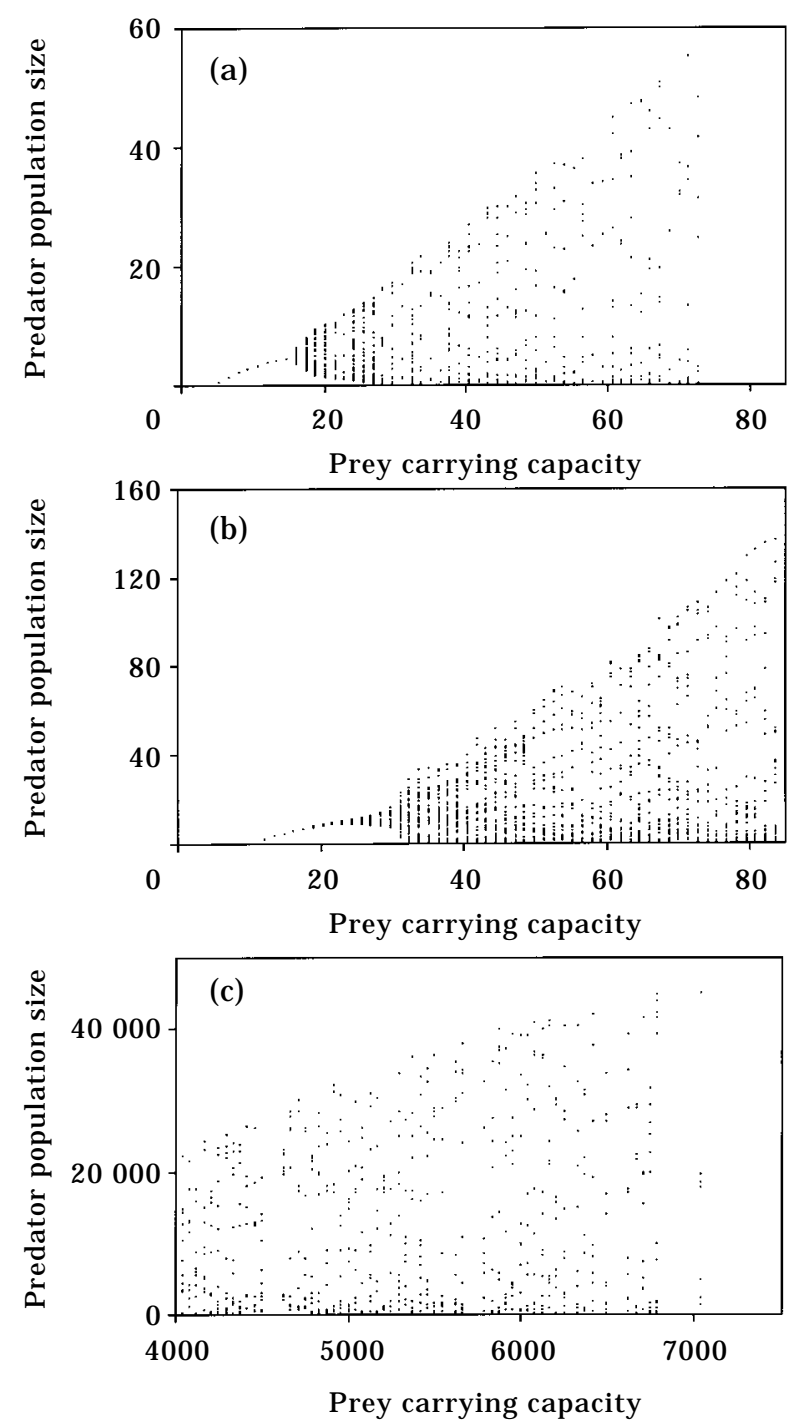

FIG. 7. Paradox of enrichment in the monomorphic [Fig. 7(a)] and in the polymorphic model [Figs 7(b) and (c)]. In each panel, for 60 equally spaced values of the prey carrying capacity $K$ the system was run for 400 generations, and then the next 50 generations were plotted on the vertical axis. Only the predator population size is shown. Prey dynamics would look qualitatively similar, except that they would reach a stable equilibrium once the predator goes extinct. For the monomorphic model, a much finer resolution of the dynamics than shown in Fig. 7(a) would be possible. However, for the sake of comparison the resolution was kept as low as in the polymorphic model, for which a higher resolution would cost too much computer time. Nevertheless, it is clear that in the polymorphic model, the predator can persist for much higher prey carrying capacities than in the monomorphic model, where persistence is only possible for $K<74$. The demographic parameters in the models were $\lambda=6, a=0.2$ and $b=1$. The number of loci in the polymorphic model was 12 , and the predation niche width parameter was set to $\sigma^{2}=6$.
Qualitatively, however, Fig. 7(c) is typical and shows that even though the genetically variable system still exhibits the paradox of enrichment in the form of increasing predator-prey oscillations, persistence is greatly enhanced and occurs for a much wider range of prey carrying capacities than in the monomorphic Nicholson-Bailey model.

\section{Discussion}

The classical predator-prey model of Nicholson \& Bailey (1935) has been the starting point for many attempts to understand the mechanisms that promote persistence in predator-prey systems. Most notably, it has been argued that interference among the predators and spatial aggregation of the predator on areas of high prey density can stabilize the interactions (Hassell \& May, 1973; Hassell, 1978; Chesson \& Murdoch, 1986). In addition, it has been shown that coexistence is possible in spatially extended systems despite local extinctions (Allen, 1975; Hassell et al., 1991; Holt \& Hassell, 1993). Somewhat analogously, I argued here that coexistence is possible between phenotypically variable predator and prey populations despite the interactions between single phenotypes being unstable. I extended the Nicholson-Bailey model (1) by assuming that the predation efficiency is determined by quantitative characters in the prey and the predator. I used a model with multiple diploid loci with additive effects to describe the genetics of this character in both populations. The genetic model used here is the diploid extension of the multi-locus models of Doebeli (1996a, b, c).

The basic results are summarized in Figs 2-6, which illustrate the general fact that, compared with the monomorphic Nicholson-Bailey model, persistence of the predator-prey interactions is greatly enhanced in the genetically variable systems. This is also true if self-limitation of the prey is included in the models, in which case both the monomorphic and the polymorphic models exhibit the paradox of enrichment, because the systems become more unstable as the prey carrying capacity is increased. However, predator-prey coexistence is possible in the polymorphic system for much higher prey carrying capacities than in the monomorphic model (Fig. 7).

If the populations persist, the predator is "tracking" the prey in phenotype space: because predators are most efficient on prey with the same character value, the mean of the character distribution in the predator always moves towards the mean in the prey, which in turn is trying to "escape" from the predator mean. This coevolutionary arms 
race between the prey and the predator results in oscillations of the character means in phenotype space [Figs 2(b) and 4(b)]. Indefinite persistence of the predator-prey interactions is linked to the condition that during these oscillations, the means never come too close to the boundary of the character interval, in which case the populations would essentially become monomorphic and would go extinct as in the basic Nicholson-Bailey model. Even if costs are associated to increases (or, equivalently, to decreases) in the characters in the prey and the predator, evolutionary cycling in phenotype space is possible and leads to lower extinction probabilities or to indefinite persistence (Fig. 5). Similar coevolutionary arms races have been observed by Marrow et al. (1992) and by Dieckmann et al. (1995), although in their asexual models the cycles in phenotype space occur due to new mutants appearing in the populations, rather than to shifts in phenotype distributions built from existing genetic variation.

If one compares the coevolutionary oscillations of the character means in the ten loci example shown in Fig. 2(b) with the oscillations shown in Fig. 4(b) for a system with 15 loci, one sees that the relative size of the oscillations compared with the size of the entire character interval is larger when there are fewer loci. In other words, relative to the entire range of phenotypes, the coevolutionary arms race between predator and prey leads to larger oscillations in the characters when they are determined by fewer loci. Although simulations become very time consuming with large numbers of loci, numerical experiments suggest that this is a general phenomenon in the model considered. Thus, not only do the interactions become persistent when the number of loci is increased, but the systems also become more stable in the sense that when the character intervals are scaled to the same size, then the coevolutionary oscillations in phenotype space become smaller and remain confined to ever smaller regions in the center of the character interval. As a consequence, such coevolutionary oscillations might be difficult to detect in real systems if a large number of loci with small effects influences the characters determining the ecological interactions.

That small evolutionary oscillations in a trait can mediate persistence of predator-prey interactions has been observed by Hochberg \& Holt (1996) in a related context. The trait they considered was a proportional prey refuge in the Nicholson-Bailey model with density dependence in the prey. The prey refuge is characterized by a number $\alpha$ in the interval $[0,1]$, and $\alpha$ is assumed to depend on quantitative characters in the prey and the predator, much like the predation efficiency is assumed to depend on such characters in the model studied here. The parameter $\alpha$ has a strong influence on the ecological dynamics in the models considered by Hochberg \& Holt (1996), and these dynamics in turn influence the evolution of the refuge parameter $\alpha$. This leads to rather complicated interactions between ecological and evolutionary dynamics. One of the main conclusions of Hochberg $\&$ Holt (1996) was that evolution of the parameter $\alpha$ tended to lead to stable equilibrium population dynamics. In particular, similar to the conclusions reached here, the evolutionary dynamics of $\alpha$ tended to promote persistence of the predator-prey interactions.

However, the two approaches are conceptually quite different. While Hochberg \& Holt (1996) studied the evolution of a trait that directly influences ecological dynamics, the trait considered here-predation efficiency - does not influence the ecological dynamics, in the sense that the Nicholson-Bailey model (1) is unstable for any predation efficiency $a$, and that the predator goes extinct for all $a$, even though the time to extinction may vary. For example, the evolution of the quantitative characters determining the refuge in Hochberg \& Holt's (1996) model could be such that both characters and hence $\alpha$ reached evolutionarily stable, constant values, which induced persistence. In contrast, in the present model persistence would be impossible with constant means and a constant (mean) predation efficiency. This can be checked by artificially imposing the condition that the means remain unchanged in the numerical simulations. Thus, no particular mean character values can induce persistence in the models studied here, and instead persistence is only possible with evolutionary oscillations in the characters determining predation efficiency. In the present model it is really genetic variation for, and not a particular value of, a character determining ecological interaction that leads to the persistence of these interactions. Genetic variation allows for the evolutionary arms race, whose oscillatory "catch and escape" mechanism, rather than the particular values attained during the cycles, are essential for persistence.

In a similar vein (but without genetics and evolutionary dynamics), Bailey et al. (1962) and, in a more general setup, Chesson \& Murdoch (1986) argued that variation in the risk of predation among the prey can stabilize predator-prey interactions and lead to persistence. Such variation could occur because some prey individuals are more difficult to find than others, because the predators search some places more than others, or because different prey individuals are exposed to different predator densities. 
However, in this non-genetic setup, in which there are no dynamics for the distribution of the predation risk among the prey, the requirements for such a distribution to induce persistence are quite restrictive (Bailey et al., 1962; Chesson \& Murdoch, 1986). In particular, a (fixed) normal distribution, which would be expected if a genetic basis for predation risk were assumed without explicitly including genetic dynamics, would not lead to persistence.

In the model described in Section 2, I have assumed that the character intervals in the prey and the predator are the same, and that the character is determined by the same number of loci in both populations. The first of these assumptions is not restrictive, because equality of the characters can be obtained by rescaling. However, the second assumption clearly is restrictive and was made here for simplicity. Nevertheless, it is quite easy to introduce different numbers of loci in the prey and the predator (while keeping the character intervals the same), and numerical experiments show that the general qualitative results of this paper remain valid in this more general case. However, it seems worth mentioning one additional feature that occurs with different numbers of loci in the prey and the predator. For a given number of loci in the prey, the "tracking ability" of the predator, i.e. the accuracy with which the mean of the predator character can follow the mean in the prey, depends on the number of loci in the predator. With relatively few loci, the genetic reshuffling due to mating is a more coarse process, and the phenotype distribution of the predators cannot track the prey phenotype distribution as accurately as with a high number of loci. On the other hand, the closer the match between the phenotype distributions in the predator and the prey, the closer one is to the original Nicholson-Bailey model. Therefore, predator-prey persistence is less likely if the number of loci in the predator is much larger than the number of loci in the prey, and persistence is more likely if the number of loci in the predator is low compared to the number of loci in the prey.

This remark fits in with results obtained by Saloniemi (1993), who extended the continuous time Lotka-Volterra predator-prey model with quantitative genetics by assuming normal distributions for characters determining the predator-prey interactions. The basic Lotka-Volterra model is structurally unstable and exhibits neutrally stable predator-prey cycles. The inclusion of the genetics breaks the structural instability and either leads to stable coexistence or to extinction, and Saloniemi
(1993) argued that a high additive genetic variance of the prey character tends to promote predator-prey coexistence at a stable equilibrium.

It is easy to see with numerical simulations that if one had asexual populations, i.e. if one only had phenotypic variability in the Nicholson-Bailey model, but no genetics [hence if only the ecological equations (3-8) were used for the dynamic recursion, but not the genetic equations (9)], then there would be no predator-prey coexistence. Thus, the reshuffling process induced by mating is essential for coexistence. A similar observation was made in Doebeli \& Koella (1994). We considered a one predator-two prey model and compared the dynamics of a system in which the prey were asexual to the dynamics of a system in which the two prey phenotypes were assumed to be determined by one locus with two alleles and random mating. We showed that in the sexual system regions of coexistence and regions of stable equilibrium dynamics were larger than in the asexual system. In a similar vein, I have shown in Doebeli (1996b) that variation in a quantitative character determining host-microparasite interactions can greatly reduce the dynamic complexity and the size of density fluctuations in sexual populations. Since large fluctuations increase the probability of extinction due to chance events, this again suggests that genetic variation can promote persistence. Inasmuch as ecological persistence is tied to evolutionary persistence, this relates to Hamilton's (1982) theory for the evolution of sex, who argued that a lower variance in fitness (which in the present context translates to smaller population fluctuations) might be a reason why sexual organisms are more common than asexual organisms.

In summary, it can be expected on theoretical grounds that genetic variability in characters determining ecological interactions promotes the persistence of the interacting populations. What is needed now is good empirical evidence testing this prediction. Ideally, such evidence is obtained from experiments, e.g. by using relatively simple and short-lived organisms such as bacteria or protists. In view of recent advances in the technology of culturing, manipulating and analysing such organisms (e.g. Lenski \& Travisano, 1994; Holyoak \& Lawler, 1996), it should be possible to study the effects of genetic variation on the persistence of real ecological systems.

I thank Gerdien de Jong and an anonymous referee for helpful comments and suggestions. This research was partially supported by the Schweizerischer Nationalfonds. 


\section{REFERENCES}

Allen, J. C. (1975). Mathematical models of species interactions in time and space. Am. Nat. 109, 319-342.

Bailey, V. A., Nicholson, A. J. \& Williams, J. (1962). Interaction between hosts and parasites when some host individuals are more difficult to find than others. J. theor. Biol. 3, 1-18.

Beddington, J. R., Free, C. A. \& Lawton, J. H. (1975). Dynamic complexity in predator-prey models framed in difference equations. Nature 255, 58-60.

Chesson, P. L. \& Murdoch, W. W. (1986). Aggregation of risk: relationships among host-parsitoid models. Am. Nat. 127, 696-715.

Dieckmann, U., Marrow, P. \& Law, R. (1995). Evolutionary cycling in predator-prey interactions-population dynamics and the red queen. J. theor. Biol. 176, 91-102.

Doebeli, M. (1996a). An explicit genetic model for ecological character displacement. Ecology 77, 510-520.

Doebeli, M. (1996b). Quantitative genetics and population dynamics. Evolution 50, 532-546.

DoEBELI, M. (1996c). A quantitative genetic competition model for sympatric speciation. J. Evol. Biol. 9, 893-909.

Doebeli, M. \& Koella, J. C. (1994). Sex and population dynamics. Proc. R. Soc. Lond. B 257, 17-23.

Edelstein-Keshet, L. (1987). Mathematical Models in Biology. New York: Random House.

Hamilton, W. D. (1982). Sex versus non-sex versus parasite. Oikos 35, 282-290.

Hassell, M. P. (1978). The Dynamics of Arthropod Predator-prey Systems. Princeton: Princeton University Press.

Hassell, M. P. \& May, R. M. (1973). Stability in insect host-parasitoid models. J. Anin. Ecol. 42, 249-262.

Hassell, M. P., Comins, H. N. \& May, R. M. (1991). Spatial structure and chaos in insect population dynamics. Nature 353, 255-258.

HochberG, M. E. \& Holt, R. D. (1995). Refuge evolution and the population dynamics of coupled host-parasitoid associations. Evol. Ecol. 9, 633-661.

Holt, R. D. \& Hassell, M. P. (1993). Environmental heterogeneity and the stability of host-parasitoid interactions. J. Anin. Ecol. 62, 89-100.

Holyoak, M. \& Lawler, S. P. (1996). Persistence of an extinction-prone predator-prey interaction through metapopulation dynamics. Ecology 77, 1867-1879.

HufFAKer, C. B. (1958). Experimental studies on predation: dispersion factors and predator-prey oscillations. Hilgardia 27, 343-383.

Huffaker, C. B., Shea, K. P. \& Herman, S. G. (1963). Experimental studies on predation. Hilgardia 34, 305-330.

Hutchinson, G. E. (1961). The paradox of the plankton. Am. Nat. 95, 137-145.

Lenski, R. E. \& Travisano, M. (1994). Dynamics of adaptation and diversification - a 10000 generation experiment with bacterial populations. Proc. Natl. Acad. Sci. U.S.A. 91, 6808-6814.

MarRow, P., LAw, R. \& CANnings, C. (1992). The coevolution of predator-prey interactions-ESSS and red queen. Proc. R. Soc. Lond. B 250, 133-141.

Murray, J. D. (1993). Mathematical Biology. 2nd Edn, Berlin: Springer Verlag.

Nicholson, A. J. \& Bailey, V. A. (1935). The balance of animal populations. Proc. Zool. Soc. Lond. 3, 551-598.

RosenzWeig, M. L. (1971). Paradox of enrichment: destabilization of exploitation ecosystems in ecological time. Science 171, 385-387.

SAloniemi, I. (1993). A coevolutionary predator-prey model with quantitative characters. Am. Nat. 141, 880-896. 